\title{
Snippets of Data at a Glance: Using RSS to Deliver Statistics
}

\section{Introduction}

For most researchers, more data are always better. The Federal Reserve Board, like other statistical institutions, has a longstanding tradition of publishing tables of data in statistical releases and has developed applications to aid users in downloading large quantities of data. For some number watchers, especially in the economic and financial realm, an observation or two is all that is needed-but it is needed the moment it is available. How can data providers serve these clients as well as those who want every observation? Our answer: provide statistics as RSS feeds for simple, immediate viewing of individual observations, publish tables and serve data via applications.

In developing mechanisms for delivering statistical content to the public from our website, we have noticed a recurring theme: the electronic information environment is shifting so that the presentation of our information is far less important than it once was. New methods of disseminating and repurposing information mean that we have less control over the appearance of our data and news than we have had in the past.

Emphasis on instant access to information on a variety of devices means that fewer of our customers are content to wait until we have information posted on a website where it will look good in a traditional browser. In response to these changes, the Federal Reserve Board, in concert with the Federal Reserve Bank of New York and several other central banks, have created RSS-CB, a technical specification for representing common central bank data.

\section{"Problems" to be Solved}

The proliferation of gadgets that deliver information means typical delivery methods are no longer sufficient. Cell phones, Blackberrys, and PDAs present new challenges for delivering information; users want their content delivered to them rather than having to go get it. And the new hardware on which they want the content has new software with which to access the information.

In addition, it is becoming more and more common for our content to be "harvested" or accessed by automated processes. The Federal Reserve Board's Data Download
San Cannon*

Program (DDP) was designed specifically to allow this type of access: interested users can write programs to download our data without ever opening a browser.

Not all users are interested in downloading a spreadsheet with just one or two numbers. These users are likely to have "screen scraping" programs to pull the latest exchange rate or commercial paper rate from an HTML table. This an inefficient method of aggregating such information and we have little control over what information is chosen or how it is used and attributed once it is extracted from our website.

The fact that our electronic content is being processed by machines and not just read by humans highlights another challenge: how to address the needs of two different audiences. Data management staff at the Board too often have to extract individual data observations from PDF files and therefore are acutely aware of this dichotomy.

Traditional methods of providing data meet the needs of one group or the other. Formatted HTML tables that humans readily understand are not the best format for machine processing of data. The Board uses SDMX as a download format for data from the public website which is easily managed by automated processes but difficult for humans to consume.

\section{First Step: An Alternative Format for the Human Readers}

While it is likely that modifying the solution for one audience would have better served the other, we decided to take a different approach and look at RSS as a format that could more easily be adapted to both retain some control and fit two different sets of consumers.

The Board joined with the Federal Reserve Bank of New York and other central banks and central banking organizations to investigate the plausibility of using RSS to represent data. Initially, these institutions were the Bank of Canada, the Banco de México, and the Bank for International Settlements. The European Central Bank (ECB) and Swiss National Bank then joined the project. This group is now organized as the Central Bank Online Communication group (CBOC). 
As the most common use of RSS is to deliver news stories, RSS might not seem to be an appropriate tool to deliver statistics, as it is not immediately clear how the elements that so readily accommodate news dissemination could represent data. It is relatively straightforward to determine what the title of a news story should be, but it is not at all straightforward to discern what title a data observation should have, or whether it even makes sense to title such an item. For example, an exchange rate has many components, including base currency, target currency, time of the observation, and the value itself. What might the title be?

The group created recommended formats for RSS representations of common central bank data. To continue with exchange rates as an example, the group recommended that the title comprise, in order, a code for the country producing the rate, units of a target currency, a target currency code, units of a base currency, a base currency code, a date, an institutional identifier, and a rate name - US: $10.6925 \mathrm{MXN}=1$ USD 2008-03-28 NYFed noon buying. This title allows a human reader to take in an exchange rate at a glance, no matter what central bank was the source. A Banco de México publication of their rate appeared as $M X: 10.6957 M X N=1 U S D$ 2008-03-28 BM For payment

The group compiled an application guide for data feeds that outlines the details for order of the elements, specification details, and issues to consider. For example, implementers of RSS-CB feeds should be aware that many RSS readers, including Live Bookmarks, have a 50 character limit so publishing exchange rates with lengthy rate names or many decimal places may not provide the best information for that observation.

\section{Second Step: A Standard for the Machines}

While specifying the details for the title allows human users to be able to consume a single observation in a Live Bookmark or RSS reader page, it doesn't make automated processing much easier if machines still have to parse text strings to obtain the pertinent information.

The group also created atomic metadata elements for the components of data that central banks report, to be used in common by the group's different members. To that end, the group decided to use RSS 1.0 as the "flavour" of RSS on which to base the standard. RSS 2.0 is more widely used owing to the simplicity of its implementation but the complexity of extending the model made it unsuitable for our purposes.

Each piece of information in the title has a corresponding atomic element, drawn from an existing metadata standard where possible and created as an extension where the existing standards were not appropriate. The clarity of the tags for the atomic elements helps to increase the likelihood that the information will not be misunderstood or misrepresented when repurposed by aggregators or other users. For example, the intended use of the value in an element called $<\mathrm{cb}$ : targetCurrency $>$ should be clear to users programming the automated processes.

Elements created as particular to central bank information are in the RSS-CB namespace and are spelled out in the standard. These elements, like the target currency example above, are identified by cb indicating that they are in the $\mathrm{cb}$, or central bank, namespace. Elements from the Dublin Core namespace (dc) or the Dublin Core terms namespace (dcterms) are also allowed and are in some cases required by the specification. Use of Dublin Core elements without specific reference in the specification is also allowed. The specification also provides for institution-specific extensions but to date no institution using RSS-CB has developed any such extensions.

The application guides and user guidelines written for the standard also give examples and requirements for the inclusion and specification of atomic elements and, where applicable, their relationship to the information displayed in the title. For example, the application guide for statistics spells out how to specify the institutional abbreviation in an interest rate feed:

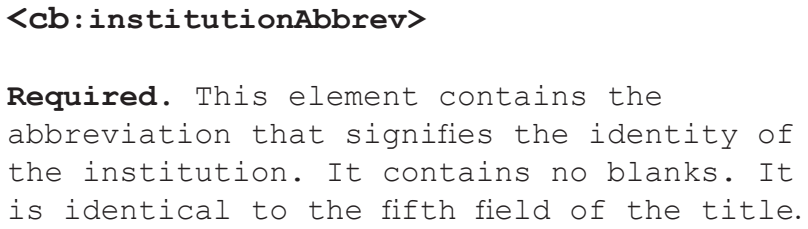

And the feed for the overnight nonfinancial commercial paper rate from the Board follows that specification where FRB is the institutional identifier for the Federal Reserve Board.

US: CP 2.24 2008-04-07 FRB Overnight AA nonfinancial commercial paper rate

For exchange rates the recommendations in the application guide are slightly different:

$<c b$ : institutionAbbrev>

Required This element contains the abbreviation that signifies the identity of the institution. It contains no blanks. It is identical to the seventh field of the title.

The previously mentioned exchange rate examples show that the New York Fed and the Banco de México have the institutional abbreviations NYFed and BM, respectively. 


\section{The Result: RSS-CB}

To continue with the exchange rate example, here are the RSS-CB elements for the NY Fed exchange rate example:

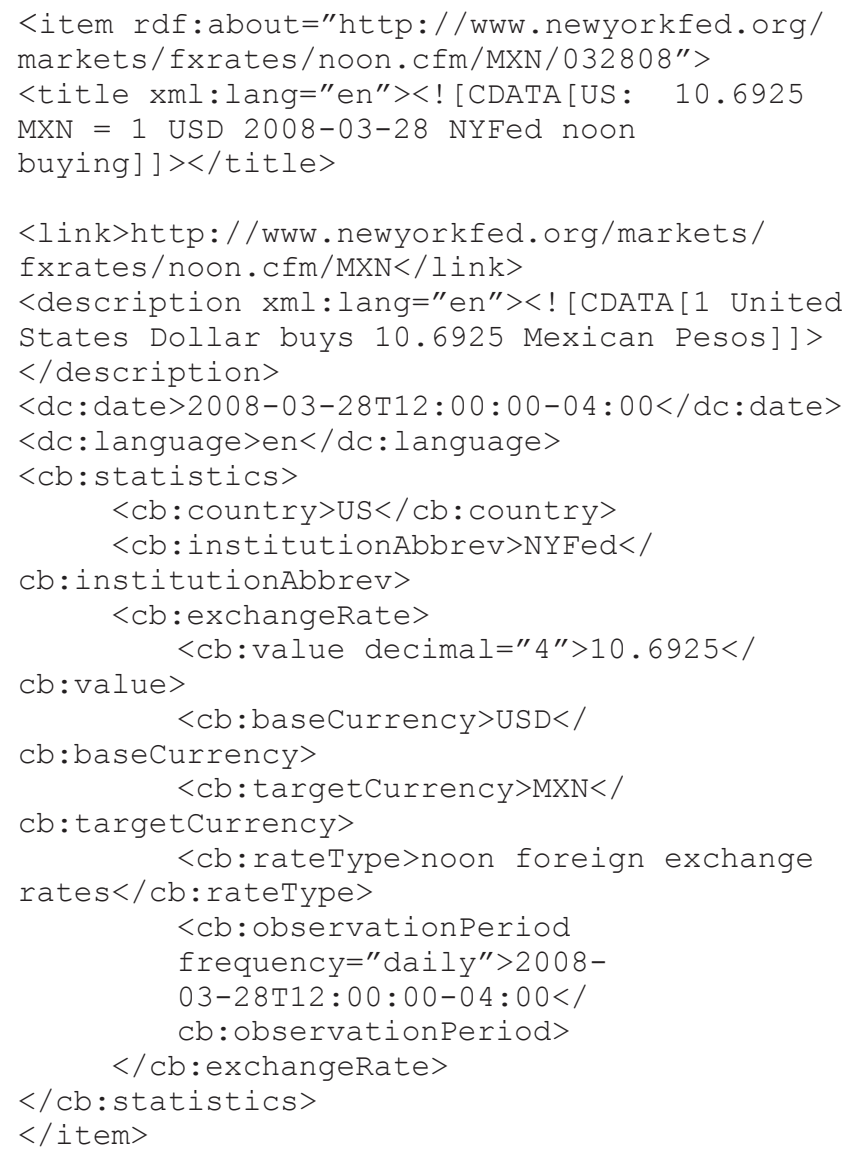

For the automated process, the information to be collected is clearly identified and easily repurposed. Such transparency helps central banks to retain some control over their information by reducing the probability of error.

For the human consumer, the title appears in their RSS reader of choice and they can discern the value of the exchange rate between the U.S. dollar and the Mexican peso at a quick glance without needing to download any files. In a Google Reader window, a list of such exchange rates from the New York Fed appears as such:

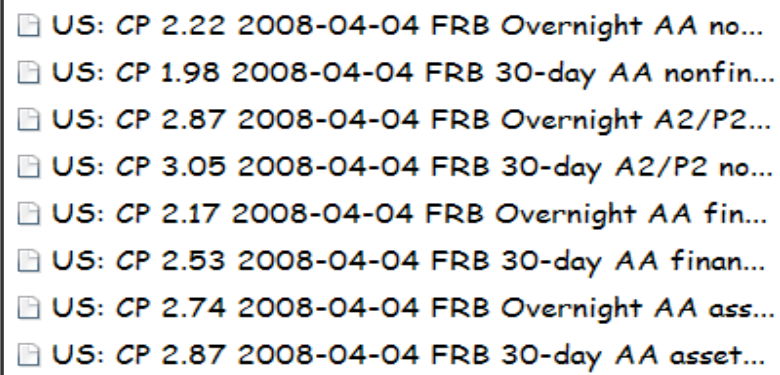

It is straightforward to see the information you want quickly. This particular feed is for all the exchange rates published by the New York Fed but single rate feeds have also been created. Users can choose to subscribe to the same rate but published by different institutions and differences will be easy to discern in such a display.

Subscribing to special reader services is not necessary to consume this information, although they are very popular. RSS-CB data feeds can be displayed in a Live Bookmark in Firefox or IE 7 such as these commercial paper rates from the Board's feed. Notice the effect of the character limit on the rate name.

Our users can add a feed to their My Yahoo! Page which has no character limit so the rate names are displayed in full and the information appears with other information in which they are interested.

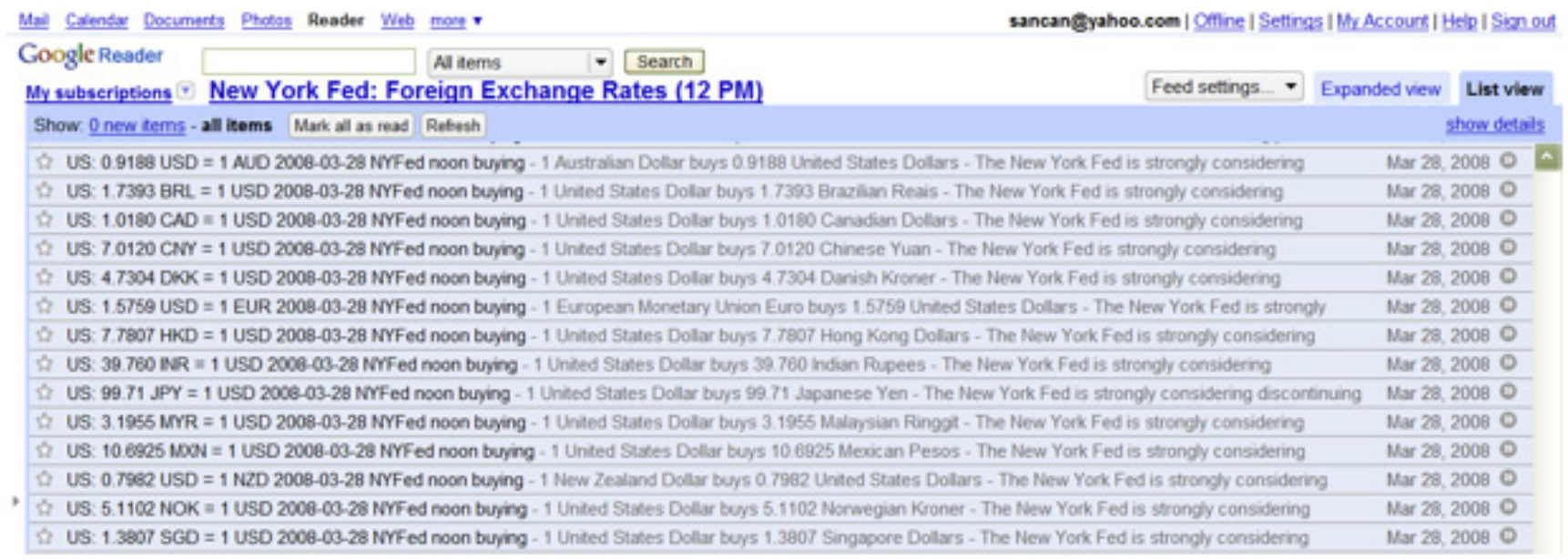




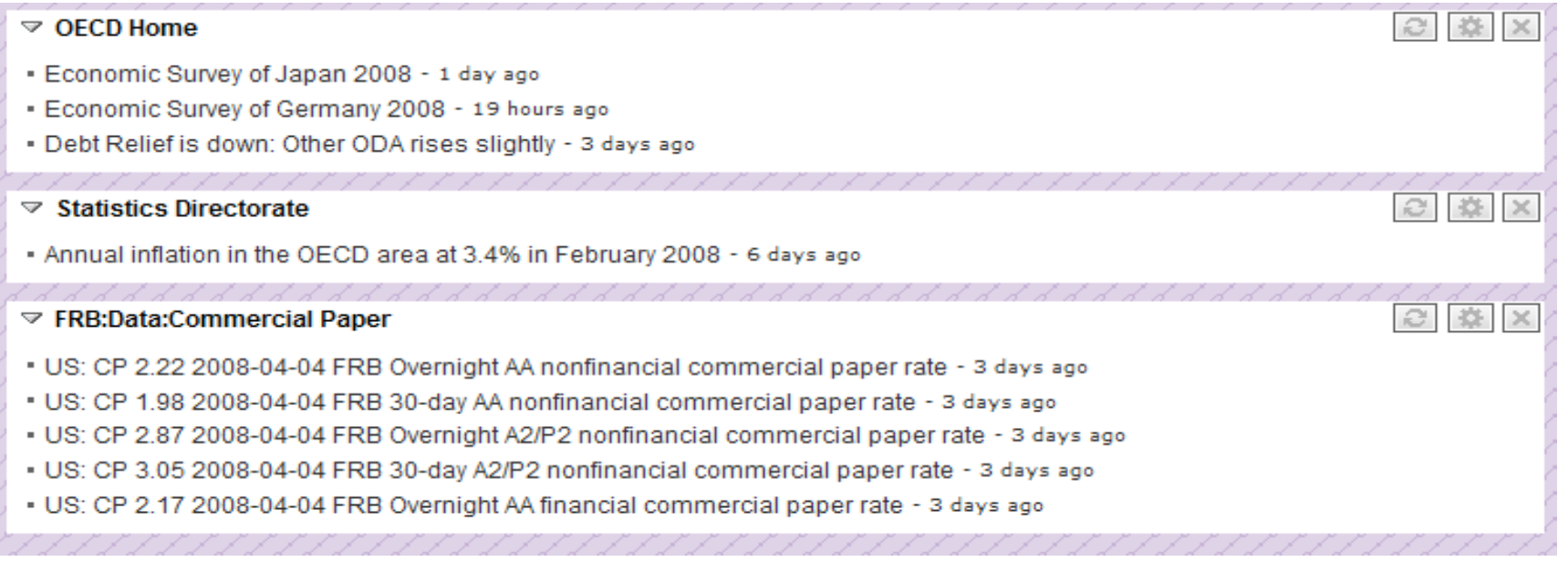

\section{STATUS AND FUTURE PLANS}

The RSS-CB standard is now on version 1.1 and is fairly stable. There are a few recent changes and developments that are being incorporated into all aspects of the specification and supporting schemas and some existing RSS-CB feeds will need to be updated to validate with the current specification.

There are several central banks besides the original developers of RSS-CB who currently publish data feeds using the specification. Some institutions adhere more closely to the standard than others. This is an unordered list of institutions that produce RSS-CB feeds, with urls of the locations of those feeds.

Banco de México (http://www.banxico.org. $\mathrm{mx} /$ sitioingles/RSS/SuscripcionRSS.html)

Bank of Canada (http://www. bankofcanada.ca/en/rates/rss_fx.html)

Bank of Finland (http://www.bof.fi/ en/suomen_pankki/ajankohtaista/muut_ uutiset/2007/uutinen_27092007.htm)

Bank for International Settlements (http://www.bis.org/rss/index.htm)

Bank Negara Malaysia (http://www. bnm.gov.my/index.php?ch=107)

European Central Bank (http://www.ecb.int/ stats/exchange/eurofxref/html/index.en.html)

Federal Reserve Bank of New York (http://www.newyorkfed.org/rss/)

Federal Reserve Board (http:// www.federalreserve.gov/feeds/)

Swiss National Bank (http://www. snb.ch/en/ifor/media/id/media_rss)

Reserve Bank of Australia (http://www.rba. gov.au/RSS/rss_cb_exchange_rates.xml)
The Central Bank Online Communications (CBOC) group meets annually as well as collaborating online at www. cbwiki.net; the details of the specification can also be found on the website. The first meeting was in October 2007 at the New York Fed and included many of the institutions listed above as well as others who participated in our discussions on how best to deliver RSS and to whom we are grateful. These additional institutions include the Centro de Estudios Monetarios Latinoamericanos (CEMLA), the Reserve Bank of Australia, the Deutsche Bundesbank, the Reserve Bank of India, the Reserve Bank of New Zealand, the Central Bank of Nigeria, the Bangko Sentral ng Pilipinas, and the Monetary Authority of Singapore. The next meeting will be at the Banco de México in October 2008 where we will continue the newly begun discussions for work on version 1.2.

\section{REFERENCES}

RSS 1.0. (2001). RDF Site Summary (RSS) 1.0. Retrieved January 23, 2008, from http://web.resource.org/rss/1.0/spec.

RSS-CB Application Guides. (2008). Application Guides. Located at http://www.cbwiki.net/wiki/index.php/ Application_Guides.

RSS-CB Specification. (2008). Specification 1.1. Located at http://www.cbwiki.net/wiki/index.php/Specification_1.1.

RSS-CB User Guide. (2008). User Guide 1.1. Located at http://www.cbwiki.net/wiki/index.php/User_guide_1.1.

\footnotetext{
* Prepared for the United Nations Economic Commission for Europe's Dissemination and Communication Work Session, Geneva, Switzerland, on May 12, 2008. An early version of this paper was presented at IASSIST in 2007. San Cannon, Chief, Economic Information Management, Federal Reserve Board, Washington DC, USA. Contact: scannon@frb.gov. The opinions are of the author and not the Federal Reserve Board.
} 